\title{
Two-Point Iterative Methods for Solving Quadratic Equations and its Applications
}

\author{
Kalyanasundaram Madhu* \\ (Communicated by İ. Onur KIYMAZ)
}

\begin{abstract}
Kung-Traub conjecture states that an iterative method without memory for locating the zero of a scalar equation could achieve convergence order $2^{d-1}$, where $d$ is the total number of function evaluations, but proposed algorithm produces convergence order of $r+2$, where $r$ is a positive integer with three function evaluations for solving quadratic equations, which is better than expected maximum convergence order. Therefore, we show that the conjecture fails for quadratic equations. Also, we extend proposed algorithm to solving systems which involving quadratic equations. We test our methods with some numerical experiments including application to one dimensional and two dimensional Bratu problems.

Keywords: Quadratic equation; Two-step iterative methods; Kung-Traub's conjecture; Efficiency Index; Systems of equations. AMS Subject Classification (2010): Primary: 65H05; 65D05

${ }^{*}$ Corresponding author
\end{abstract}

\section{Introduction}

It is accepted that a boundary value problems among theory of gases, elasticity and different applied area are for the most part reduced in one variable nonlinear equations. The zeros of nonlinear equations is also real or complex. Generally, there are two eccentric of technique acting offered to seek out the zeros of scalar nonlinear equations. First, direct technique, that don't seem to be continually applicable to seek out the roots and second, iterative technique acting supported the idea of consecutive approximations. In this case, the ultimate procedure is to begin out with one or additional initial approximations to the zero and attain a sequence of iterates whose the limit converges to the reality solution. Multipoint iterative methods for locating nonlinear equations are of nice sensible importance since they overcome theoretical limits of one-point methods concerning the order of convergence and computational potency. Here, we have a tendency to specializing in finding the simple root of nonlinear equations by an iterative method.

One of the simplest root-finding strategies is Newton's iteration methodology for finding a nonlinear equation. The order of convergence of Newton's methodology 2 and it's optimal with two function evaluations per iterative step. In recent years, varied higher order iterative methods are developed and analyzed for finding nonlinear equations that improve classical strategies similar to Newton's, Euler's, Chebyshev-Halley's methods, etc. Recently, the order of convergence of the many variants of Newton's methodology has been improved victimisation constant range of functional evaluations by suggests that of weight functions $[2,4,5,12,13,15,16,19,20]$. The aim of such analysis is to develop best methods that satisfy Kung-Traub's conjecture [13]. Conjecture says that the order of convergence of any multi-point method without memory with $d$ evaluations cannot exceed the bound $2^{d-1}$, the optimal order.

Let $x^{(k+1)}=\psi\left(x^{(k)}\right)$ define an Iterative Function (I.F.).

Received : 12-04-2018, Accepted : 24-10-2018 
Definition 1.1. [22] If the sequence $\left\{x^{(k)}\right\}$ tends to a limit $x^{*}$ in such a way that

$$
\lim _{n \rightarrow \infty} \frac{x^{(k+1)}-x^{*}}{\left(x^{(k)}-x^{*}\right)^{p}}=C
$$

for $p \geq 1$, then the order of convergence of the sequence is said to be $p$, and $C$ is known as the asymptotic error constant. If $p=1, p=2$ or $p=3$, the convergence is said to be linear, quadratic or cubic, respectively.

Let $\varepsilon^{(k)}=x^{(k)}-x^{*}$, then the relation

$$
\varepsilon^{(k+1)}=C\left(\varepsilon^{(k)}\right)^{p}+O\left(\varepsilon^{(k)}\right)^{p+1}=O\left(\varepsilon^{(k)}\right)^{p} .
$$

is called the error equation. The value of $p$ is called the order of convergence of the method.

Definition 1.2. [18] The Efficiency Index is given by

$$
E I=p^{\frac{1}{d}},
$$

where $d$ is the total number of new function evaluations per iteration.

Let $x^{(k+1)}$ be determined by new information at $x^{(k)}, \phi_{1}\left(x^{(k)}\right), \ldots, \phi_{\mathrm{i}}\left(x^{(k)}\right), \mathrm{i} \geq 1$.

No old information is reused. Thus,

$$
x^{(k+1)}=\psi\left(x^{(k)}, \phi_{1}\left(x^{(k)}\right), \ldots, \phi_{\mathrm{i}}\left(x^{(k)}\right)\right) .
$$

Then $\psi$ is called a multi-point I.F without memory.

Kung-Traub's Conjecture [13]

Let $\psi$ be an I.F. without memory with $d$ evaluations. Then

$$
p(\psi) \leq p_{\text {opt }}=2^{d-1},
$$

where $p_{\text {opt }}$ is the maximum order.

The second order Newton I.F. $\left(2^{n d} N R\right)$ is given by

$$
\psi_{2^{n d} N R}(x)=x-n(x), n(x)=\frac{f(x)}{f^{\prime}(x)} .
$$

The $2^{\text {nd }} N R$ I.F. is satisfies the Kung-Traub conjecture with $d=2$. Thus, $E I_{2^{n d} N R}=1.414$. Let us consider two-step third order I.F. $\left(3^{r d} \mathrm{CM}\right)[7]$ is given by

$$
\psi_{3^{r d} C M}(x)=x-\frac{1}{2}(3-\eta(x)) n(x), \eta(x)=\frac{f^{\prime}\left(x-\frac{2}{3} n(x)\right)}{f^{\prime}(x)} .
$$

The $3^{r d} C M$ I.F. with three function evaluations and it is not satisfies the conjecture. According to conjecture, not possible to derive an I.F. without memory more than order four with three function evaluations. Recently, Ahmad [1] and Babajee [3] developed two-step eighth order methods using weight functions, also both are showed Kung-Traub's conjecture is fails for solving quadratic equations. They termed as $8^{\text {th }} B Q I M$ and it is given below

$$
\psi_{(8)^{t h} B Q I M}(x)=x-H(\eta(x), 6) n(x),
$$

where $H(\eta(x), 6)=1-\frac{3}{4}(\eta(x)-1)+\frac{9}{8}(\eta(x)-1)^{2}-\frac{135}{64}(\eta(x)-1)^{3}+\frac{567}{128}(\eta(x)-1)^{4}-\frac{5103}{512}(\eta(x)-1)^{5}+\frac{24057}{1024}(\eta(x)-1)^{6}$. Thukral [21], demonstrated that the Kung and Traub conjecture fails for a particular case, that is when the simple root of a nonlinear equation is equal to zero. Madhu [14], author developed two-step iterative methods with three function evaluations reaching convergence order $r+3$, where $r$ is a positive integer. In this paper, we develop two-step higher order iterative methods for solving quadratic equations and study Kung-Traub's conjecture for proposed methods.

The rest of the paper is organized as follows. Section 2 presents the development of the new methods and its convergence analysis for scalar equation. In section 3, we extend these methods to systems of quadratic equations and derived its convergence analysis. Section 4 carries out the tests on the numerical examples and compare the present methods with Newton's method and $8^{\text {th }} B Q I M$. Section 5 gives application on 1-D and 2-D bratu problems. Finally, section 6 gives conclusions on our work. 


\section{Developments of the methods}

Let us consider the proposed scheme as,

$$
\psi_{(r+2)^{t h} K M}(x)=x-\frac{1}{2}(3-\eta(x)) H(\eta(x), r) n(x),
$$

where

$$
H(\eta(x), r)=1+\sum_{i=1}^{r} a_{i}(\eta(x)-1)^{i},
$$

where $a_{i}{ }^{\prime}$ s are constants. If $r=6$, then the error equation of the I.F. (2.1) is as follows

$$
\begin{aligned}
\psi(x)- & x^{*}=\frac{1}{3}\left(c_{2}+4 c_{2} a_{1}\right)\left(\varepsilon^{(k)}\right)^{2}-\frac{2}{9}\left(c_{2}^{2}\left(-3+20 a_{1}+8 a_{2}\right)\right)\left(\varepsilon^{(k)}\right)^{3} \\
& +\frac{2}{27} c_{2}^{3}\left(-63+150 a_{1}+152 a_{2}+32 a_{3}\right)\left(\varepsilon^{(k)}\right)^{4} \\
& -\frac{4}{81}\left(c_{2}^{4}\left(-351+432 a_{1}+948 a_{2}+448 a_{3}+64 a_{4}\right)\right)\left(\varepsilon^{(k)}\right)^{5} \\
& +\frac{8}{243} c_{2}^{5}\left(729 a_{1}+2\left(-810+2367 a_{2}+1920 a_{3}+592 a_{4}+64 a_{5}\right)\right)\left(\varepsilon^{(k)}\right)^{6} \\
& +\frac{16}{729} c_{2}^{6}\left(1701 a_{1}-2\left(10017 a_{2}+2\left(-1701+6408 a_{3}+3216 a_{4}+736 a_{5}+64 a_{6}\right)\right)\right)\left(\varepsilon^{(k)}\right)^{7} \\
& -\frac{32}{2187}\left(c_{2}^{7}\left(26973+25515 a_{1}-72009 a_{2}-145098 a_{3}-106272 a_{4}-38688 a_{5}-7040 a_{6}\right)\right)\left(\varepsilon^{(k)}\right)^{8} \\
& +O\left(\varepsilon^{(k)}\right)^{9}
\end{aligned}
$$

where $c_{2}=f^{\prime \prime}\left(x^{*}\right) / 2 f^{\prime}\left(x^{*}\right)$.

Eliminating the terms in $\left(\varepsilon^{(k)}\right)^{j}, j=2,3,4,5,6,7$ and we obtain the following system of linear equations

$$
\begin{aligned}
& \frac{4}{3} a_{1}=-\frac{1}{3}, \\
& -\frac{40}{9} a_{1}-\frac{16}{9} a_{2}=-\frac{2}{3}, \\
& \frac{300}{27} a_{1}+\frac{304}{27} a_{2}+\frac{64}{27} a_{3}=\frac{126}{27}, \\
& \frac{300}{27} a_{1}+\frac{304}{27} a_{2}+\frac{64}{27} a_{3}=\frac{126}{27}, \\
& -\frac{1728}{21} a_{1}-\frac{3792}{81} a_{2}-\frac{1792}{81} a_{3}-\frac{256}{81} a_{4}=-\frac{1404}{88}, \\
& \frac{5832}{243} a_{1}+\frac{3782}{243} a_{2}+\frac{37200}{243} a_{3}+\frac{9472}{243} a_{4}+\frac{1024}{243} a_{5}=\frac{12960}{243}, \\
& \frac{27216}{729} a_{1}-\frac{320544}{729} a_{2}-\frac{410112}{729} a_{3}-\frac{205824}{729} a_{4}-\frac{4104}{729} a_{5}-\frac{4096}{729} a_{6}=-\frac{108864}{729} .
\end{aligned}
$$

The solutions of system of linear equation can obtained easily from first equation. The solutions are $a_{1}=-\frac{1}{4}, a_{2}=1$, $a_{3}=-\frac{103}{64}, a_{4}=\frac{29}{8}, a_{5}=-\frac{4175}{512}$ and $a_{6}=\frac{9941}{512}$.

In this approach, we tend to get a family of higher order I.F.s $\left((r+2)^{n d} K M\right)$ for solving quadratic equations. Consider first six members of proposed family (2.1) with their error equation,

1. $r=1$ : two-step $3^{r d} K M$ I.F.

$$
\begin{gathered}
H(\eta(x), 1)=1-\frac{1}{4}(\eta(x)-1) . \\
\psi_{3^{r d} K M}(x)-x^{*}=\left(\frac{16}{9} c_{2}^{2}\right)\left(\varepsilon^{(k)}\right)^{3}+O\left(\left(\varepsilon^{(k)}\right)^{4}\right) .
\end{gathered}
$$

2. $r=2$ : two-step $4^{\text {th }} \mathrm{KM}$ I.F.

$$
\begin{gathered}
H(\eta(x), 2)=1-\frac{1}{4}(\eta(x)-1)+(\eta(x)-1)^{2} . \\
\psi_{4^{\text {th } K M}}(x)-x^{*}=\left(\frac{103}{27} c_{2}^{3}\right)\left(\varepsilon^{(k)}\right)^{4}+O\left(\left(\varepsilon^{(k)}\right)^{5}\right) .
\end{gathered}
$$


3. $r=3$ : two-step $5^{\text {th }}$ KM I.F.

$$
\begin{gathered}
H(\eta(x), 3)=1-\frac{1}{4}(\eta(x)-1)+(\eta(x)-1)^{2}-\frac{103}{64}(\eta(x)-1)^{3} . \\
\psi_{5^{t h} K M}(x)-x^{*}=\left(\frac{928}{81} c_{2}^{4}\right)\left(\varepsilon^{(k)}\right)^{5}+O\left(\left(\varepsilon^{(k)}\right)^{6}\right) .
\end{gathered}
$$

4. $r=4$ : two-step $6^{\text {th }}$ KM I.F.

$$
\begin{gathered}
H(\eta(x), 4)=1-\frac{1}{4}(\eta(x)-1)+(\eta(x)-1)^{2}-\frac{103}{64}(\eta(x)-1)^{3}+\frac{29}{8}(\eta(x)-1)^{4} . \\
\psi_{6^{\text {th } K M}}(x)-x^{*}=\left(\frac{8350}{243} c_{2}^{5}\right)\left(\varepsilon^{(k)}\right)^{6}+O\left(\left(\varepsilon^{(k)}\right)^{7}\right) .
\end{gathered}
$$

5. $r=5$ : two-step $7^{\text {th }}$ KM I.F.

$$
\begin{gathered}
H(\eta(x), 5)=1-\frac{1}{4}(\eta(x)-1)+(\eta(x)-1)^{2}-\frac{103}{64}(\eta(x)-1)^{3}+\frac{29}{8}(\eta(x)-1)^{4}-\frac{4175}{512}(\eta(x)-1)^{5} . \\
\psi_{7^{\text {th }} K M}(x)-x^{*}=\left(\frac{79528}{729} c_{2}^{6}\right)\left(\varepsilon^{(k)}\right)^{7}+O\left(\left(\varepsilon^{(k)}\right)^{8}\right) .
\end{gathered}
$$

6. $r=6$ : two-step $8^{\text {th }}$ KM I.F.

$$
\begin{gathered}
H(\eta(x), 6)=1-\frac{1}{4}(\eta(x)-1)+(\eta(x)-1)^{2}-\frac{103}{64}(\eta(x)-1)^{3}+\frac{29}{8}(\eta(x)-1)^{4}-\frac{4175}{512}(\eta(x)-1)^{5}+\frac{9941}{512}(\eta(x)-1)^{6} . \\
\psi_{8^{\text {th } K M}}(x)-x^{*}=\left(\frac{779167}{2187} c_{2}^{7}\right)\left(\varepsilon^{(k)}\right)^{8}+O\left(\left(\varepsilon^{(k)}\right)^{9}\right) .
\end{gathered}
$$

Note that we've got obtained associate eighth order with only three function evaluations for solving quadratic equations.

\subsection{Convergence Analysis}

Theorem 2.1. Let a sufficiently smooth function $f: D \subset \mathbb{R} \rightarrow \mathbb{R}$ has a simple root $x^{*}$ in the open interval $D$. Then the six members of two-step $(r+2)^{r d} \mathrm{KM}^{\prime}$ s family in $(2.1)(r=1,2,3,4,5,6)$ are of local third to eighth order convergence, respectively.

Proof. We will prove the third and eighth order convergence, fourth to seventh order I.F.s follow on similar lines. It's straightforward to examine that for a quadratic function,

$$
f(x)=f^{\prime}\left(x^{*}\right)\left[\varepsilon^{(k)}+c_{2}\left(\varepsilon^{(k)}\right)^{2}\right]
$$

and

$$
f^{\prime}(x)=f^{\prime}\left(x^{*}\right)\left[1+2 c_{2} \varepsilon^{(k)}\right] .
$$

By Taylor expansion and using computer algebra software MATHEMATICA

$$
\begin{aligned}
n(x) & =\varepsilon^{(k)}-c_{2}\left(\varepsilon^{(k)}\right)^{2}+2 c_{2}{ }^{2}\left(\varepsilon^{(k)}\right)^{3}-4 c_{2}{ }^{3}\left(\varepsilon^{(k)}\right)^{4}+8 c_{2}^{4}\left(\varepsilon^{(k)}\right)^{5}-16 c_{2}{ }^{5}\left(\varepsilon^{(k)}\right)^{6} \\
& +32 c_{2}{ }^{6}\left(\varepsilon^{(k)}\right)^{7}-64 c_{2}{ }^{7}\left(\varepsilon^{(k)}\right)^{8}+128 c_{2}{ }^{8}\left(\varepsilon^{(k)}\right)^{9}+\ldots
\end{aligned}
$$

so that

$$
\begin{aligned}
\eta(x)=1 & -\frac{4}{3} c_{2} \varepsilon^{(k)}+4 c_{2}{ }^{2}\left(\varepsilon^{(k)}\right)^{2}-\frac{32}{3} c_{2}{ }^{3}\left(\varepsilon^{(k)}\right)^{3}+\frac{80}{3} c_{2}{ }^{4}\left(\varepsilon^{(k)}\right)^{4}-64 c_{2}{ }^{5}\left(\varepsilon^{(k)}\right)^{5}+\frac{448}{3} c_{2}{ }^{6}\left(\varepsilon^{(k)}\right)^{6} \\
& -\frac{1024}{3} c_{2}{ }^{7}\left(\varepsilon^{(k)}\right)^{7}+768 c_{2}{ }^{8}\left(\varepsilon^{(k)}\right)^{8}+\ldots
\end{aligned}
$$


Now,

$$
H(\eta(x), 1)=1+c_{2} \varepsilon^{(k)}-3 c_{2}^{2}\left(\varepsilon^{(k)}\right)^{2}+8 c_{2}^{3}\left(\varepsilon^{(k)}\right)^{3}+\ldots .
$$

Using eqs. (2.2), (2.3) and (2.4), we have

$$
\frac{1}{2}(3-\eta(x)) H(\eta(x), 1) n(x)=\varepsilon^{(k)}-\left(\frac{16}{9} c_{2}^{2}\right)\left(\varepsilon^{(k)}\right)^{3}+O\left(\left(\varepsilon^{(k)}\right)^{4}\right),
$$

which leads to the error equation for the two-step $3^{\text {rd }}$ KM I.F.

Similarly,

$$
H(\eta(x), 6)=1+c_{2} \varepsilon^{(k)}-c_{2}{ }^{2}\left(\varepsilon^{(k)}\right)^{2}+c_{2}{ }^{3}\left(\varepsilon^{(k)}\right)^{3}-c_{2}{ }^{4}\left(\varepsilon^{(k)}\right)^{4}+c_{2}{ }^{5}\left(\varepsilon^{(k)}\right)^{5}-c_{2}{ }^{6}\left(\varepsilon^{(k)}\right)^{6}-428 c_{2}{ }^{7}\left(\varepsilon^{(k)}\right)^{7}+\ldots .
$$

Using eqs. (2.2) and (2.5), we have

$$
\frac{1}{2}(3-\eta(x)) H(\eta(x), 6) n(x)=\varepsilon^{(k)}-\left(\frac{779167}{2187} c_{2}^{7}\right)\left(\varepsilon^{(k)}\right)^{8}+O\left(\left(\varepsilon^{(k)}\right)^{9}\right),
$$

which leads to the error equation for the two-step $8^{\text {th }}$ KM I.F.

We next prove the local convergence of the two-step $(r+2)^{n d} K M^{\prime}$ s family for any $r$.

Theorem 2.2. Let a sufficiently smooth function $f: D \subset \mathbb{R} \rightarrow \mathbb{R}$ has a simple root $x^{*}$ in the open interval $D$. Then the members of two-step $(r+2)^{\text {nd }} \mathrm{KM}^{\prime}$ s family in (2.1) are of local $r+2$ order convergence.

Proof. We prove this result by induction. The case $r=1$ corresponds to the $3^{r d} K M$ I.F. Assume the two-step $(r+2)^{t h} K M$ family has order of convergence of $(r+2)$. Then it satisfies the error equation

$$
\psi_{(r+2)^{t h} K M}(x)-x^{*}=C_{r} c_{2}^{r+1}\left(\varepsilon^{(k)}\right)^{r+2}+O\left(\left(\varepsilon^{(k)}\right)^{r+3}\right),
$$

where $C_{r}$ is the asymptotic error constant. Assume that eq. (2.6) holds for $r=m$. Now from eq (2.3), we have

$$
\eta(x)-1=-\frac{4}{3} c_{2} \varepsilon^{(k)}\left(1-3 c_{2} \varepsilon^{(k)}+8 c_{2}^{2}\left(\varepsilon^{(k)}\right)^{2}+\ldots\right),
$$

so that

$$
\begin{aligned}
(\eta(x)-1)^{m+1} & =\left(-\frac{4}{3}\right)^{m+1} c_{2}{ }^{m+1}\left(\varepsilon^{(k)}\right)^{m+1}\left(1-3 c_{2} \varepsilon^{(k)}+8 c_{2}{ }^{2}\left(\varepsilon^{(k)}\right)^{2}+\ldots\right)^{m+1} \\
& =\left(-\frac{4}{3}\right)^{m+1} c_{2}{ }^{m+1}\left(\varepsilon^{(k)}\right)^{m+1}\left(1+O\left(\varepsilon^{(k)}\right)\right) .
\end{aligned}
$$

For the case $r=m+1$,

$$
\begin{aligned}
& \psi_{(m+3)^{r d} K M}(x)-x^{*} \\
& =x-\frac{1}{2}(3-\eta(x)) n(x) H(\eta(x), m+1)-x^{*} \\
& =x-\frac{1}{2}(3-\eta(x)) n(x) H(\eta(x), m)-x^{*}-a_{m+1} \frac{1}{2}(3-\eta(x)) n(x)(\eta(x)-1)^{m+1} \\
& =\psi_{(m+2)^{t h} K M}(x)-x^{*}-a_{m+1} \frac{1}{2}(3-\eta(x)) n(x)(\eta(x)-1)^{m+1} \\
& =C_{m} c_{2}{ }^{m+1}\left(\varepsilon^{(k)}\right)^{m+2}-a_{m+1}\left(-\frac{4}{3}\right)^{m+1} c_{2}{ }^{m+1}\left(\varepsilon^{(k)}\right)^{m+2}+O\left(\left(\varepsilon^{(k)}\right)^{m+3}\right) \text { using eqs. (2.2), (2.3), (2.6) and (2.7), } \\
& =\left(C_{m}-a_{m+1}\left(-\frac{4}{3}\right)^{m+1}\right) c_{2}{ }^{m+1}\left(\varepsilon^{(k)}\right)^{m+2}+O\left(\left(\varepsilon^{(k)}\right)^{m+3}\right)
\end{aligned}
$$

which shows that the two-step $(m+3)^{r d} \mathrm{KM}$ family has $(m+3)$ rd order of convergence if we choose

$$
a_{m+1}=C_{m}\left(-\frac{3}{4}\right)^{m+1} \text {. }
$$


From eq. (2.8), we can obtain higher order I.F. if we know the asymptotic error constant of the previous I.F. For example, for the two-step $3^{r d}$ KM I.F., $C_{1}=\frac{16}{9}$ and from eq. (2.8),

$$
a_{2}=C_{1}\left(-\frac{3}{4}\right)^{2}=1
$$

and we can obtain the $4^{\text {th }}$ KM I.F. Similarly, for the two-step $8^{\text {th }}$ KM I.F., $C_{6}=\frac{779167}{2184}$ and from eq. (2.8),

$$
a_{7}=C_{6}\left(-\frac{3}{4}\right)^{7}=-\frac{779167}{16384}
$$

and we can obtain the two-step $9^{\text {th }}$ KM I.F. with

$$
\begin{aligned}
H(\eta(x), 7) & =1-\frac{1}{4}(\eta(x)-1)+(\eta(x)-1)^{2}-\frac{103}{64}(\eta(x)-1)^{3}+\frac{29}{8}(\eta(x)-1)^{4}-\frac{4175}{512}(\eta(x)-1)^{5} \\
& +\frac{9941}{512}(\eta(x)-1)^{6}-\frac{779167}{16384}(\eta(x)-1)^{7} .
\end{aligned}
$$

From Theorem 2.2, we conclude that we can have a family of order $r+2, r=1,2, \ldots$ with only 3 function evaluations.

Remark: The Efficiency Index of the two-step $(r+2)^{t h} \mathrm{KM}$ family is

$$
E I=(r+2)^{\frac{1}{3}}, r \geq 1 .
$$

Hence, we conclude that this is the one having highest efficiency index. Next section, we extend proposed methods to systems.

\section{Extension to systems of equations}

Let us consider, system of nonlinear equations $\mathbf{f}(\mathbf{x})=\mathbf{0}$, where $\mathbf{f}(\mathbf{x})=\left(f_{1}(\mathbf{x}), f_{2}(\mathbf{x}), \ldots, f_{n}(\mathbf{x})\right)^{T}, \mathbf{x}=\left(x_{1}, x_{2}, \ldots, x_{n}\right)^{T}$, $f_{i}: \mathbb{R}^{n} \rightarrow \mathbb{R}, \forall i=1,2, \ldots, n$ defined as

$$
f_{i}(\mathbf{x})=b_{i}+\sum_{l=1}^{n} \sum_{m=1}^{n} b_{l, m} x_{l} x_{m}, \quad b_{i}, b_{l, m}, i, l, m=1, . . n, \text { are constants. }
$$

and $\mathbf{f}: \mathbf{D} \subset \mathbb{R}^{n} \rightarrow \mathbb{R}^{n}$ is a smooth map and $\mathbf{D}$ is an open and convex set, where we assume that $\mathbf{x}^{*}=\left(x_{1}^{*}, x_{2}^{*}, \ldots, x_{n}^{*}\right)^{T}$ is a root of the system and $\mathbf{x}^{(0)}=\left(x_{1}^{(0)}, x_{2}^{(0)}, \ldots, x_{n}^{(0)}\right)^{T}$ is an initial point sufficiently close to $\mathbf{x}^{*}$. Let us define the two-step $(r+2)^{t h} K M^{\prime}$ 's family for systems of quadratic equations as:

$$
\psi_{(r+2)^{t h} K M}(\mathbf{x})=\mathbf{x}-\frac{1}{2}(3-\eta(\mathbf{x})) \mathbf{H}(\eta(\mathbf{x}), r) \mathbf{n}(\mathbf{x})
$$

where

$$
\begin{aligned}
\mathbf{n}(\mathbf{x}) & =\mathbf{f}^{\prime}(\mathbf{x})^{-1} \mathbf{f}(\mathbf{x}) \\
\mathbf{y}(\mathbf{x}) & =\mathbf{x}-\frac{2}{3} \mathbf{n}(\mathbf{x}) \\
\eta(\mathbf{x}) & =\mathbf{f}^{\prime}(\mathbf{x})^{-1} \mathbf{f}^{\prime}(\mathbf{y}(\mathbf{x})) \\
\mathbf{H}(\eta(\mathbf{x}), r) & =\mathbf{I}+\sum_{i=1}^{r} a_{i}(\eta(\mathbf{x})-\mathbf{I})^{i}, \quad \mathbf{I} \text { is the identity matrix. }
\end{aligned}
$$

Let us define

$$
\mathbf{c}_{2}=\frac{1}{2}\left[\mathbf{f}^{\prime}\left(\mathbf{x}^{*}\right)\right]^{-1} \mathbf{f}^{(2)}\left(\mathbf{x}^{*}\right), \epsilon^{(k)}=\mathbf{x}^{(k)}-\mathbf{x}^{*} .
$$

Using the notations in [9], it is noted that $\mathbf{c}_{2} \epsilon^{(k)} \in \mathcal{L}\left(\mathbb{R}^{n}\right)$. The error at the $(k+1)$ th iteration is $\epsilon^{(k+1)}=\mathbf{L}\left(\epsilon^{(k)}\right)^{p}+$ $O\left(\left(\epsilon^{(k)}\right)^{p+1}\right)$, where $\mathbf{L}$ is a $p$-linear function $\mathbf{L} \in \mathcal{L}\left(\mathbb{R}^{n} \times \cdots \times \mathbb{R}^{n}, \mathbb{R}^{n}\right)$, is called the error equation and $p$ is the order of convergence. Observe that $\left(\epsilon^{(k)}\right)^{p}$ is $\left(\epsilon^{(k)}, \epsilon^{(k)}, \cdots, \epsilon^{(k)}\right)$. The first 6 members of $(r+2)^{t h} K M^{\prime}$ s family in (3.1) with their error equation are 
1. $r=1$ : two-step $3^{r d} K M$ I.F.

$$
\begin{gathered}
H(\eta(\mathbf{x}), 1)=\mathbf{I}-\frac{1}{4}(\eta(\mathbf{x})-\mathbf{I}) . \\
\psi_{3^{t h} K M}(\mathbf{x})-\mathbf{x}^{*}=\left(\frac{16}{9} \mathbf{c}_{2}^{2}\right)\left(\epsilon^{(k)}\right)^{3}+O\left(\left(\epsilon^{(k)}\right)^{4}\right) .
\end{gathered}
$$

2. $r=2$ : two-step $4^{\text {th }} \mathrm{KM}$ I.F.

$$
\begin{gathered}
H(\eta(\mathbf{x}), 2)=\mathbf{I}-\frac{1}{4}(\eta(\mathbf{x})-\mathbf{I})+(\eta(\mathbf{x})-\mathbf{I})^{2} . \\
\psi_{4^{t h} K M}(\mathbf{x})-\mathbf{x}^{*}=\left(\frac{103}{27} \mathbf{c}_{2}{ }^{3}\right)\left(\epsilon^{(k)}\right)^{4}+O\left(\left(\epsilon^{(k)}\right)^{5}\right) .
\end{gathered}
$$

3. $r=3$ : two-step $5^{\text {th }} \mathrm{KM}$ I.F.

$$
\begin{gathered}
H(\eta(\mathbf{x}), 3)=\mathbf{I}-\frac{1}{4}(\eta(\mathbf{x})-\mathbf{I})+(\eta(\mathbf{x})-\mathbf{I})^{2}-\frac{103}{64}(\eta(\mathbf{x})-\mathbf{I})^{3} . \\
\psi_{5^{t h} K M}(\mathbf{x})-\mathbf{x}^{*}=\left(\frac{928}{81} \mathbf{c}_{2}{ }^{4}\right)\left(\epsilon^{(k)}\right)^{5}+O\left(\left(\epsilon^{(k)}\right)^{6}\right) .
\end{gathered}
$$

4. $r=4$ : two-step $6^{\text {th }} \mathrm{KM}$ I.F.

$$
\begin{gathered}
H(\eta(\mathbf{x}), 4)=\mathbf{I}-\frac{1}{4}(\eta(\mathbf{x})-\mathbf{I})+(\eta(\mathbf{x})-\mathbf{I})^{2}-\frac{103}{64}(\eta(\mathbf{x})-\mathbf{I})^{3}+\frac{29}{8}(\eta(\mathbf{x})-\mathbf{I})^{4} . \\
\psi_{6^{t h} K M}(\mathbf{x})-\mathbf{x}^{*}=\left(\frac{8350}{243} \mathbf{c}_{2}^{5}\right)\left(\epsilon^{(k)}\right)^{6}+O\left(\left(\epsilon^{(k)}\right)^{7}\right) .
\end{gathered}
$$

5. $r=5$ : two-step $7^{\text {th }} \mathrm{KM}$ I.F.

$$
\begin{gathered}
H(\eta(\mathbf{x}), 5)=\mathbf{I}-\frac{1}{4}(\eta(\mathbf{x})-\mathbf{I})+(\eta(\mathbf{x})-\mathbf{I})^{2}-\frac{103}{64}(\eta(\mathbf{x})-\mathbf{I})^{3}+\frac{29}{8}(\eta(\mathbf{x})-\mathbf{I})^{4}-\frac{4175}{512}(\eta(\mathbf{x})-\mathbf{I})^{5} . \\
\psi_{7^{\text {th }} K M}(\mathbf{x})-\mathbf{x}^{*}=\left(\frac{79528}{729} \mathbf{c}_{2}{ }^{6}\right)\left(\epsilon^{(k)}\right)^{7}+O\left(\left(\epsilon^{(k)}\right)^{8}\right) .
\end{gathered}
$$

6. $r=6$ : two-step $8^{t h} K M$ I.F.

$$
\begin{aligned}
\mathbf{H}(\eta(\mathbf{x}), 6)= & \mathbf{I}-\frac{1}{4}(\eta(\mathbf{x})-\mathbf{I})+(\eta(\mathbf{x})-\mathbf{I})^{2}-\frac{103}{64}(\eta(\mathbf{x})-\mathbf{I})^{3}+\frac{29}{8}(\eta(\mathbf{x})-\mathbf{I})^{4}-\frac{4175}{512}(\eta(\mathbf{x})-\mathbf{I})^{5} \\
& +\frac{9941}{512}(\eta(\mathbf{x})-\mathbf{I})^{6} . \\
& \psi_{8^{t h} K M}(\mathbf{x})-\mathbf{x}^{*}=\left(\frac{779167}{2187} \mathbf{c}_{2}^{7}\right)\left(\epsilon^{(k)}\right)^{8}+O\left(\left(\epsilon^{(k)}\right)^{9}\right) .
\end{aligned}
$$

\subsection{Convergence Analysis}

Theorem 3.1. Let $\mathbf{f}: \mathbf{D} \subseteq \mathbb{R}^{n} \longrightarrow \mathbb{R}^{n}$ be twice Frechet differentiable at each point of an open convex neighborhood $\mathbf{D}$ of $\mathbf{x}^{*} \in \mathbb{R}^{n}$, that is a solution of the quadratic system $\mathbf{f}(\mathbf{x})=\mathbf{0}$. Let us suppose that $\mathbf{f}^{\prime}(\mathbf{x})$ is continuous and nonsingular in $\mathbf{x}^{*}$, and $\mathbf{x}^{(0)}$ is close enough to $\mathbf{x}^{*}$. Then the sequence $\left\{\mathbf{x}^{(k)}\right\}_{k \geq 0}$ obtained using the iterative expressions $(3.1), r=1,2, \ldots, 6$ converge to $\mathbf{x}^{*}$ with order 3 to 8 , respectively.

\section{Proof:}

We prove now for $r=6$ case. The other cases follows similar lines. Since $\mathbf{f}$ is a quadratic function of several variables, we have

and

$$
\mathbf{f}\left(\mathbf{x}^{(k)}\right)=\mathbf{f}^{\prime}\left(\mathbf{x}^{*}\right)\left[\epsilon^{(k)}+\mathbf{c}_{2}\left(\epsilon^{(k)}\right)^{2}\right]
$$

$$
\mathbf{f}^{\prime}\left(\mathbf{x}^{(k)}\right)=\mathbf{f}^{\prime}\left(\mathbf{x}^{*}\right)\left[\mathbf{I}+2 \mathbf{c}_{2} \epsilon^{(k)}\right]
$$




$$
\begin{aligned}
\mathbf{f}^{\prime}\left(\mathbf{x}^{(k)}\right)^{-1}= & {\left[\mathbf{I}-2 \mathbf{c}_{2} \epsilon^{(k)}+4 \mathbf{c}_{2}{ }^{2}\left(\epsilon^{(k)}\right)^{2}-8 \mathbf{c}_{2}{ }^{3}\left(\epsilon^{(k)}\right)^{3}+16 \mathbf{c}_{2}{ }^{4}\left(\epsilon^{(k)}\right)^{4}-32 \mathbf{c}_{2}{ }^{5}\left(\epsilon^{(k)}\right)^{5}\right.} \\
& \left.+64 \mathbf{c}_{2}{ }^{6}\left(\epsilon^{(k)}\right)^{6}-128 \mathbf{c}_{2}{ }^{7}\left(\epsilon^{(k)}\right)^{7}+256 \mathbf{c}_{2}{ }^{8}\left(\epsilon^{(k)}\right)^{8} \ldots\right]\left[\mathbf{f}^{\prime}\left(\mathbf{x}^{*}\right)\right]^{-1}
\end{aligned}
$$

Using eq. (3.2) and (3.4), we have

$$
\begin{aligned}
\mathbf{u}\left(\mathbf{x}^{(k)}\right)=\epsilon^{(k)}-\mathbf{c}_{2}\left(\epsilon^{(k)}\right)^{2}+2 \mathbf{c}_{2}{ }^{2}\left(\epsilon^{(k)}\right)^{3}-4 \mathbf{c}_{2}{ }^{3}\left(\epsilon^{(k)}\right)^{4}+8 \mathbf{c}_{2}{ }^{4}\left(\epsilon^{(k)}\right)^{5}-16 \mathbf{c}_{2}{ }^{5}\left(\epsilon^{(k)}\right)^{6} \\
+32 \mathbf{c}_{2}{ }^{6}\left(\epsilon^{(k)}\right)^{7}-64 \mathbf{c}_{2}{ }^{7}\left(\epsilon^{(k)}\right)^{8}+\ldots,
\end{aligned}
$$

and the expression for $\mathbf{y}\left(\mathbf{x}^{(k)}\right)$ is given by

$$
\begin{aligned}
& \mathbf{y}\left(\mathbf{x}^{(k)}\right)= \mathbf{x}^{*} \\
&+\frac{1}{3} \epsilon^{(k)}+\frac{2}{3} \mathbf{c}_{2}\left(\epsilon^{(k)}\right)^{2}-\frac{4}{3} \mathbf{c}_{2}{ }^{2}\left(\epsilon^{(k)}\right)^{3}+\frac{8}{3} \mathbf{c}_{2}{ }^{3}\left(\epsilon^{(k)}\right)^{4}-\frac{16}{3} \mathbf{c}_{2}{ }^{4}\left(\epsilon^{(k)}\right)^{5}+\frac{32}{3} \mathbf{c}_{2}{ }^{5}\left(\epsilon^{(k)}\right)^{6} \\
&-\frac{64}{3} \mathbf{c}_{2}{ }^{6}\left(\epsilon^{(k)}\right)^{7}+\frac{128}{3} \mathbf{c}_{2}{ }^{7}\left(\epsilon^{(k)}\right)^{8}+\ldots
\end{aligned}
$$

The Taylor expansion of Jacobian matrix $\mathbf{f}^{\prime}\left(\mathbf{y}\left(\mathbf{x}^{(k)}\right)\right)$ is then given by

$$
\begin{aligned}
\mathbf{f}^{\prime}\left(\mathbf{y}\left(\mathbf{x}^{(k)}\right)\right)=\mathbf{f}^{\prime}\left(\mathbf{x}^{*}\right)[\mathbf{I}+ & \left.2 \mathbf{c}_{2}\left(\mathbf{y}\left(\mathbf{x}^{(k)}\right)-\mathbf{x}^{*}\right)\right] \\
=\mathbf{f}^{\prime}\left(\mathbf{x}^{*}\right)[\mathbf{I}+ & \frac{2}{3} \mathbf{c}_{2}\left(\epsilon^{(k)}\right)+\frac{4}{3} \mathbf{c}_{2}{ }^{2}\left(\epsilon^{(k)}\right)^{2}-\frac{8}{3} \mathbf{c}_{2}{ }^{3}\left(\epsilon^{(k)}\right)^{3}+\frac{16}{3} \mathbf{c}_{2}{ }^{4}\left(\epsilon^{(k)}\right)^{4}-\frac{32}{3} \mathbf{c}_{2}{ }^{5}\left(\epsilon^{(k)}\right)^{5} \\
& \left.+\frac{64}{3} \mathbf{c}_{2}{ }^{6}\left(\epsilon^{(k)}\right)^{6}-\frac{128}{3} \mathbf{c}_{2}{ }^{7}\left(\epsilon^{(k)}\right)^{7}+\frac{256}{3} \mathbf{c}_{2}{ }^{8}\left(\epsilon^{(k)}\right)^{8}+\ldots .\right] .
\end{aligned}
$$

Therefore, using eq. (3.4), we obtain

$$
\begin{aligned}
\eta\left(\mathbf{x}^{(k)}\right)= & {\left[\mathbf{f}^{\prime}\left(\mathbf{x}^{(k)}\right)\right]^{-1} \mathbf{f}^{\prime}\left(\mathbf{y}\left(\mathbf{x}^{(k)}\right)\right) } \\
= & \mathbf{I}-\frac{4}{3} \mathbf{c}_{2}\left(\epsilon^{(k)}\right)+4 \mathbf{c}_{2}{ }^{2}\left(\epsilon^{(k)}\right)^{2}-\frac{32}{3} \mathbf{c}_{2}{ }^{3}\left(\epsilon^{(k)}\right)^{3}+\frac{80}{3} \mathbf{c}_{2}{ }^{4}\left(\epsilon^{(k)}\right)^{4}-64 \mathbf{c}_{2}{ }^{5}\left(\epsilon^{(k)}\right)^{5} \\
& \quad+\frac{448}{3} \mathbf{c}_{2}{ }^{6}\left(\epsilon^{(k)}\right)^{6}-\frac{1024}{3} \mathbf{c}_{2}{ }^{7}\left(\epsilon^{(k)}\right)^{7}+768 \mathbf{c}_{2}{ }^{8}\left(\epsilon^{(k)}\right)^{8}+\ldots
\end{aligned}
$$

so that

$$
\begin{gathered}
\mathbf{H}(\eta(\mathbf{x}), 6)=\mathbf{I}+\mathbf{c}_{2}\left(\epsilon^{(k)}\right)-\mathbf{c}_{2}{ }^{2}\left(\epsilon^{(k)}\right)^{2}+\mathbf{c}_{2}{ }^{3}\left(\epsilon^{(k)}\right)^{3}-\mathbf{c}_{2}{ }^{4}\left(\epsilon^{(k)}\right)^{4}+\mathbf{c}_{2}{ }^{5}\left(\epsilon^{(k)}\right)^{5} \\
-\mathbf{c}_{2}{ }^{6}\left(\epsilon^{(k)}\right)^{6}-428 \mathbf{c}_{2}{ }^{7}\left(\epsilon^{(k)}\right)^{7}+\ldots . .
\end{gathered}
$$

Using eq. (3.5), (3.6) and (3.7), we have, after simplifications,

$$
\mathbf{x}-\frac{1}{2}(3-\eta(\mathbf{x})) \mathbf{H}(\eta(\mathbf{x}), 6) \mathbf{u}\left(\mathbf{x}^{(k)}\right)=\mathbf{x}^{*}+\frac{779167}{2187} \mathbf{c}_{2}{ }^{7}\left(\epsilon^{(k)}\right)^{8}+\ldots .
$$

Theorem 3.2. Let $\mathbf{f}: \mathbf{D} \subseteq \mathbb{R}^{n} \longrightarrow \mathbb{R}^{n}$ be twice Frechet differentiable at each point of an open convex neighborhood $\mathbf{D}$ of $\mathbf{x}^{*} \in \mathbb{R}^{n}$, that is a solution of the quadratic system $\mathbf{f}(\mathbf{x})=\mathbf{0}$. Let us suppose that $\mathbf{f}^{\prime}(\mathbf{x})$ is continuous and nonsingular in $\mathbf{x}^{*}$, and $\mathbf{x}^{(0)}$ is close enough to $\mathbf{x}^{*}$. Then the sequence $\left\{\mathbf{x}^{(k)}\right\}_{k \geq 0}$ obtained using the iterative expressions (3.1), $r=1,2, \ldots$ converges to $\mathrm{x}^{*}$ with order $r+2$ with the error equation

$$
\psi_{(r+2)^{t h} K M}(\mathbf{x})-\mathbf{x}^{*}=C_{r} \mathbf{c}_{2}^{r+1}\left(\epsilon^{(k)}\right)^{r+2}+\ldots
$$

The will prove by induction and follows on similar lines. Similarly as in the case of scalar, we are able to get higher order I.F. for systems if we know the asymptotic error constant of the previous I.F. using

$$
a_{r+1}=C_{r}\left(-\frac{3}{4}\right)^{r+1}, r=1,2, \ldots
$$




\section{Numerical Experiments}

\subsection{Scalar equation}

This section deals with numerical comparisons in the MATLAB computer code rounding to 1000 significant digits. The criteria to stopping used for the iterative process $\left|x^{(k+1)}-x^{(k)}\right|<10^{-50}$. The number of iterations for convergence based on criteria is denoted by $N$. The computational order of convergence $(\rho)$ for successive zero is given by

$$
\rho=\frac{\ln \mid\left(x^{(N)}-x^{(N-1)} /\left(x^{(N-1)}-x^{(N-2)}\right) \mid\right.}{\ln \left|\left(x^{(N-1)}-x^{(N-2)}\right) /\left(x^{(N-2)}-x^{(N-3)}\right)\right|} .
$$

We consider the Test problem 1 (TP1) of finding the real zero of the quadratic function $f(x)=x^{2}+4 x-1$ to compare the proposed methods. We choose $x^{(0)}=1$. The results in Table 1 shows that, the order of the $(r+2)^{n d} K M$ I.F. $(r=1,2,3,4,5,6)$, methods converge in less number of iterations. The computational order of convergence $(\rho)$ confirming that the Kung-Traub's conjecture fails for quadratic equations. By analysis of TP1, our method $8^{\text {th }} \mathrm{KM}$ is found to be superior to the methods of $2^{\text {nd }} \mathrm{NR}$ and $8^{\text {th }} \mathrm{BQIM}$.

\subsection{Systems of Nonlinear equations}

This section deals with numerical comparisons in the MATLAB computer code rounding to 1000 significant digits. The criteria to stopping used for the iterative process

$$
\left\|\mathbf{x}^{(k+1)}-\mathbf{x}^{(k)}\right\|_{2}<10^{-100} .
$$

The approximated computational order of convergence $p_{c}$ given by (see [10])

$$
p_{c} \approx \frac{\log \left(\left\|\mathbf{x}^{(k+1)}-\mathbf{x}^{(k)}\right\|_{2} /\left\|\mathbf{x}^{(k)}-\mathbf{x}^{(k-1)}\right\|_{2}\right)}{\log \left(\left\|\mathbf{x}^{(k)}-\mathbf{x}^{(k-1)}\right\|_{2} /\left\|\mathbf{x}^{(k-1)}-\mathbf{x}^{(k-2)}\right\|_{2}\right)} .
$$

We consider the Test Problem 2 (TP2)

$$
\begin{aligned}
& x_{1}^{2}+x_{2}^{2}-7=0, \\
& x_{1}-x_{2}+1=0 .
\end{aligned}
$$

Using the substitution method, eq. (4.3) reduces to the quadratic equation $x_{2}^{2}-x_{2}-3=0$ whose positive root is given by $x_{2}^{*}=\frac{1+\sqrt{13}}{2}=2.302775638$. Therefore $x_{1}^{*}=x_{2}^{*}-1=\frac{\sqrt{13}}{2}=1.302775638$. We use $\mathbf{x}^{(0)}=(1,2)^{T}$ as initial vector and apply proposed methods (3.1), $r=1,2, \ldots, 6$ to locate the approximate solutions of eq. (4.3). Table 2 shows that our method $8^{\text {th }} \mathrm{KM}$ is converge less number of iterations with least error than $2^{\text {nd }} \mathrm{NR}$ and $8^{\text {th }}$ BQIM. Hence, Proposed methods are found to be superior to the methods of $2^{\text {nd }} \mathrm{NR}$ and $8^{\text {th }} \mathrm{BQIM}$.

We next consider the Test Problem 3 (TP3) [8]

$$
\begin{aligned}
& x_{1}^{2}+x_{2}^{2}-1=0 \\
& x_{1}^{2}-x_{2}^{2}-0.5=0
\end{aligned}
$$

Equation (4.4) reduces to the simple quadratic equation $2 x_{2}^{2}-1.5=0$ by the elimination method, whose root is given by $x_{2}^{*}=\frac{\sqrt{3}}{2}=0.866025403$ and therefore $x_{1}^{*}=\frac{1}{2}$. We choose initial point far from the root, $\mathbf{x}^{(0)}=(2,3)^{T}$ and apply our methods (3.1), $r=1,2, \ldots, 6$ to locate the approximate solutions of eq. (4.4). In Table 3, we observe that the methods take more iterations to converge when initial point is far from the root. By analysis TP3 problem, our method $8^{t h} \mathrm{KM}$ is converge with least error than $2^{\text {nd }} \mathrm{NR}$ and $8^{\text {th }}$ BQIM. Therefore, proposed methods are found to be superior to the methods of $2^{\text {nd }} \mathrm{NR}$ and $8^{\text {th }} \mathrm{BQIM}$.

We next consider the Test Problem 4 (TP4)[11].

$$
\begin{aligned}
& x_{2} x_{3}+x_{4}\left(x_{2}+x_{3}\right)=0, \\
& x_{1} x_{3}+x_{4}\left(x_{1}+x_{3}\right)=0, \\
& x_{1} x_{2}+x_{4}\left(x_{1}+x_{2}\right)=0 \\
& x_{1} x_{2}+x_{1} x_{3}+x_{2} x_{3}=1
\end{aligned}
$$

Apply the substitution method in eq. (4.5) reduces to the scalar nonlinear equation $3 x_{1}^{2}-1=0$ whose real root is given by $x_{1}^{*}=\frac{1}{\sqrt{3}}=0.577350269$. Therefore $x_{2}^{*}=x_{3}^{*}=x_{1}^{*}=\frac{1}{\sqrt{3}}=0.577350269$.

and $x_{4}^{*}=-\frac{x_{1}^{*}}{2}=-\frac{1}{2 \sqrt{3}}=-0.288675134$. Using $\mathbf{x}^{(0)}=(0.5,0.5,0.5,-0.25)^{T}$ as initial point and we apply our 


\begin{tabular}{|c|c|c|c|c|c|c|c|c|}
\hline & \multicolumn{2}{|c|}{ Existing Methods } & \multicolumn{7}{|c|}{ Members of Proposed Methods } \\
\hline error & $2^{\text {nd }}$ NR & $8^{\text {th }}$ BQIM & $3^{\text {rd }} \mathrm{KM}$ & $4^{\text {th }} \mathrm{KM}$ & $5^{\text {th }} \mathrm{KM}$ & $6^{\text {th }} \mathrm{KM}$ & $7^{\text {th }} \mathrm{KM}$ & $8^{\text {th }} \mathrm{KM}$ \\
\hline$\left|x_{1}-x_{0}\right|$ & 0.6667 & 0.7638 & 0.7426 & 0.7583 & 0.7620 & 0.7633 & 0.7637 & 0.7638 \\
\hline$\left|x_{2}-x_{1}\right|$ & 0.0952 & $9.5 \mathrm{e}-005$ & 0.0214 & 0.0056 & 0.0019 & $6.4 \mathrm{e}-004$ & $2.3 \mathrm{e}-004$ & $8.5 \mathrm{e}-005$ \\
\hline$\left|x_{3}-x_{2}\right|$ & 0.0020 & $8.3 \mathrm{e}-035$ & $8.4 \mathrm{e}-007$ & $4.3 \mathrm{e}-011$ & $7.0 \mathrm{e}-016$ & $1.4 \mathrm{e}-021$ & $4.9 \mathrm{e}-028$ & $2.8 \mathrm{e}-035$ \\
\hline$\left|x_{4}-x_{3}\right|$ & $9.1 \mathrm{e}-007$ & $2.8 \mathrm{e}-275$ & $5.4 \mathrm{e}-020$ & $1.4 \mathrm{e}-043$ & $5.0 \mathrm{e}-078$ & $1.6 \mathrm{e}-127$ & $1.0 \mathrm{e}-193$ & $4.0 \mathrm{e}-279$ \\
\hline$\left|x_{5}-x_{4}\right|$ & $1.8 \mathrm{e}-013$ & - & $1.4 \mathrm{e}-059$ & $1.9 \mathrm{e}-173$ & - & - & - & - \\
\hline$\left|x_{6}-x_{5}\right|$ & $7.9 \mathrm{e}-027$ & - & - & - & - & - & - & - \\
\hline$\left|x_{7}-x_{6}\right|$ & $1.4 \mathrm{e}-053$ & - & - & - & - & - & - & - \\
\hline$\rho$ & 1.99 & 7.99 & 2.99 & 3.99 & 4.99 & 5.99 & 6.99 & 7.99 \\
\hline
\end{tabular}

Table 1. Results of TP1 for the $2^{n d} \mathrm{NR}, 8^{\text {th }}$ BQIM with new families of I.F.s $(\mathrm{r}=1,2,3,4,5,6)$

\begin{tabular}{|c|c|c|c|c|c|c|c|c|}
\hline & \multicolumn{2}{|c|}{ Existing Methods } & \multicolumn{7}{|c|}{ Members of Proposed Methods } \\
\hline error & $2^{\text {nd }} \mathrm{NR}$ & $8^{t h}$ BQIM & $3^{\text {rd }} \mathrm{KM}$ & $4^{\text {th }} \mathrm{KM}$ & $5^{\text {th } \mathrm{KM}}$ & $6^{\text {th } \mathrm{KM}}$ & $7^{\text {th } \mathrm{KM}}$ & $8^{\text {th } \mathrm{KM}}$ \\
\hline$\left\|\mathbf{x}^{(1)}-\mathbf{x}^{(0)}\right\|_{2}$ & 0.4714 & 0.4282 & 0.4203 & 0.4299 & 0.4276 & 0.4284 & 0.4281 & 0.4282 \\
\hline$\left\|\mathbf{x}^{(2)}-\mathbf{x}^{(1)}\right\|_{2}$ & 0.0429 & $3.0 \mathrm{e}-005$ & 0.0079 & 0.0017 & $5.7 \mathrm{e}-004$ & $1.8 \mathrm{e}-004$ & $6.5 \mathrm{e}-005$ & $2.3 \mathrm{e}-005$ \\
\hline$\left\|\mathbf{x}^{(3)}-\mathbf{x}^{(2)}\right\|_{2}$ & $3.6 \mathrm{e}-004$ & $3.9 \mathrm{e}-039$ & $3.3 \mathrm{e}-008$ & $2.4 \mathrm{e}-013$ & $1.0 \mathrm{e}-018$ & $4.4 \mathrm{e}-025$ & $3.3 \mathrm{e}-032$ & $4.0 \mathrm{e}-040$ \\
\hline$\left\|\mathbf{x}^{(4)}-\mathbf{x}^{(3)}\right\|_{2}$ & $2.5 \mathrm{e}-008$ & $3.0 \mathrm{e}-310$ & $2.5 \mathrm{e}-024$ & $1.0 \mathrm{e}-052$ & $2.2 \mathrm{e}-092$ & $7.4 \mathrm{e}-149$ & $2.8 \mathrm{e}-223$ & $2.6 \mathrm{e}-318$ \\
\hline$\left\|\mathbf{x}^{(5)}-\mathbf{x}^{(4)}\right\|_{2}$ & $1.2 \mathrm{e}-016$ & - & $1.1 \mathrm{e}-072$ & $3.4 \mathrm{e}-210$ & 0 & - & - & - \\
\hline$\left\|\mathbf{x}^{(6)}-\mathbf{x}^{(5)}\right\|_{2}$ & $3.1 \mathrm{e}-033$ & - & $1.1 \mathrm{e}-217$ & - & - & - & - & - \\
\hline$\left\|\mathbf{x}^{(7)}-\mathbf{x}^{(6)}\right\|_{2}$ & $1.9 \mathrm{e}-066$ & - & - & - & - & - & - & - \\
\hline$\left\|\mathbf{x}^{(8)}-\mathbf{x}^{(7)}\right\|_{2}$ & $7.4 \mathrm{e}-133$ & - & - & - & - & - & - & - \\
\hline$p_{c}$ & 2.00 & 8.12 & 3 & 3.99 & 5 & 6.14 & 7.16 & 8.16 \\
\hline
\end{tabular}

Table 2. Results of TP2 for the $2^{n d} \mathrm{NR}, 8^{\text {th }}$ BQIM with new families of I.F.s $(\mathrm{r}=1,2,3,4,5,6)$

\begin{tabular}{|c|c|c|c|c|c|c|c|c|}
\hline & \multicolumn{7}{|c|}{ Existing Methods } & \multicolumn{7}{|c|}{ Members of Proposed Methods } \\
\hline error & $2^{\text {nd }} \mathrm{NR}$ & $8^{\text {th }}$ BQIM & $3^{\text {rd }} \mathrm{KM}$ & $4^{\text {th }} \mathrm{KM}$ & $5^{\text {th }} \mathrm{KM}$ & $6^{\text {th }} \mathrm{KM}$ & $7^{\text {th }} \mathrm{KM}$ & $8^{\text {th }} \mathrm{KM}$ \\
\hline$\left\|\mathbf{x}^{(1)}-\mathbf{x}^{(0)}\right\|_{2}$ & 1.6694 & 2.5146 & 2.0799 & 2.2689 & 2.3649 & 2.4334 & 2.4824 & 2.5197 \\
\hline$\left\|\mathbf{x}^{(2)}-\mathbf{x}^{(1)}\right\|_{2}$ & 0.7437 & 0.2477 & 0.5974 & 0.4729 & 0.3931 & 0.3291 & 0.2805 & 0.2424 \\
\hline$\left\|\mathbf{x}^{(3)}-\mathbf{x}^{(2)}\right\|_{2}$ & 0.2824 & $1.5 \mathrm{e}-004$ & 0.0883 & 0.0242 & 0.0076 & 0.0021 & $5.5 \mathrm{e}-004$ & $1.2 \mathrm{e}-004$ \\
\hline$\left\|\mathbf{x}^{(4)}-\mathbf{x}^{(3)}\right\|_{2}$ & 0.0681 & $1.7 \mathrm{e}-028$ & $8.9 \mathrm{e}-004$ & $1.1 \mathrm{e}-006$ & $2.7 \mathrm{e}-010$ & $3.1 \mathrm{e}-015$ & $1.7 \mathrm{e}-021$ & $2.7 \mathrm{e}-029$ \\
\hline$\left\|\mathbf{x}^{(5)}-\mathbf{x}^{(4)}\right\|_{2}$ & 0.0046 & $3.9 \mathrm{e}-220$ & $1.2 \mathrm{e}-009$ & $6.1 \mathrm{e}-024$ & $1.8 \mathrm{e}-047$ & $3.3 \mathrm{e}-086$ & $4.4 \mathrm{e}-144$ & $1.3 \mathrm{e}-226$ \\
\hline$\left\|\mathbf{x}^{(6)}-\mathbf{x}^{(5)}\right\|_{2}$ & $2.1 \mathrm{e}-005$ & - & $3.6 \mathrm{e}-027$ & $5.4 \mathrm{e}-093$ & $2.4 \mathrm{e}-233$ & 0 & - & - \\
\hline$\left\|\mathbf{x}^{(7)}-\mathbf{x}^{(6)}\right\|_{2}$ & $4.4 \mathrm{e}-010$ & - & $8.4 \mathrm{e}-080$ & 0 & - & - & - & - \\
\hline$\left\|\mathbf{x}^{(8)}-\mathbf{x}^{(7)}\right\|_{2}$ & $1.9 \mathrm{e}-019$ & - & $1.0 \mathrm{e}-237$ & - & - & - & - & - \\
\hline$\left\|\mathbf{x}^{(9)}-\mathbf{x}^{(8)}\right\|_{2}$ & $3.9 \mathrm{e}-038$ & - & - & - & - & - & - & - \\
\hline$\left\|\mathbf{x}^{(10)}-\mathbf{x}^{(9)}\right\|_{2}$ & $1.5 \mathrm{e}-075$ & - & - & - & - & - & - & - \\
\hline$\left\|\mathbf{x}^{(11)}-\mathbf{x}^{(10)}\right\|_{2}$ & $2.3 \mathrm{e}-150$ & - & - & - & - & - & - & - \\
\hline$p_{c}$ & 2.00 & 7.50 & 3.00 & 4.00 & 4.99 & 6.00 & 6.98 & 7.60 \\
\hline
\end{tabular}

Table 3. Results of TP3 for the $2^{\text {nd }} \mathrm{NR}$, $8^{\text {th }}$ BQIM with new families of I.F.s $(r=1,2,3,4,5,6)$ 


\begin{tabular}{|c|c|c|c|c|c|c|c|c|}
\hline & \multicolumn{2}{|c|}{ Existing Methods } & \multicolumn{7}{|c|}{ Members of Proposed Methods } \\
\hline error & $2^{\text {nd }} \mathrm{NR}$ & $8^{\text {th }}$ BQIM & $3^{\text {rd }} \mathrm{KM}$ & $4^{\text {th }} \mathrm{KM}$ & $5^{\text {th }} \mathrm{KM}$ & $6^{\text {th } \mathrm{KM}}$ & $7^{\text {th }} \mathrm{KM}$ & $8^{\text {th } \mathrm{KM}}$ \\
\hline$\left\|\mathbf{x}^{(1)}-\mathbf{x}^{(0)}\right\|_{2}$ & 0.1502 & 0.1394 & 0.1379 & 0.1397 & 0.1394 & 0.1395 & 0.1394 & 0.1394 \\
\hline$\left\|\mathbf{x}^{(2)}-\mathbf{x}^{(1)}\right\|_{2}$ & 0.0107 & $1.4 \mathrm{e}-006$ & 0.0015 & $2.5 \mathrm{e}-004$ & $6.2 \mathrm{e}-005$ & $1.5 \mathrm{e}-005$ & $4.0 \mathrm{e}-006$ & $1.1 \mathrm{e}-006$ \\
\hline$\left\|\mathbf{x}^{(3)}-\mathbf{x}^{(2)}\right\|_{2}$ & $5.5 \mathrm{e}-005$ & $3.9 \mathrm{e}-047$ & $1.3 \mathrm{e}-009$ & $1.6 \mathrm{e}-015$ & $5.9 \mathrm{e}-022$ & $1.2 \mathrm{e}-029$ & $2.5 \mathrm{e}-038$ & $4.6 \mathrm{e}-048$ \\
\hline$\left\|\mathbf{x}^{(4)}-\mathbf{x}^{(3)}\right\|_{2}$ & $1.4 \mathrm{e}-009$ & 0 & $1.1 \mathrm{e}-027$ & $3.2 \mathrm{e}-060$ & $4.7 \mathrm{e}-107$ & $2.9 \mathrm{e}-174$ & $8.6 \mathrm{e}-264$ & 0 \\
\hline$\left\|\mathbf{x}^{(5)}-\mathbf{x}^{(4)}\right\|_{2}$ & $1.0 \mathrm{e}-018$ & - & $5.5 \mathrm{e}-082$ & $4.4 \mathrm{e}-239$ & - & - & - & - \\
\hline$\left\|\mathbf{x}^{(6)}-\mathbf{x}^{(5)}\right\|_{2}$ & $5.1 \mathrm{e}-037$ & - & $7.1 \mathrm{e}-245$ & - & - & - & - & - \\
\hline$\left\|\mathbf{x}^{(7)}-\mathbf{x}^{(6)}\right\|_{2}$ & $1.2 \mathrm{e}-073$ & - & - & - & - & - & - & - \\
\hline$\left\|\mathbf{x}^{(8)}-\mathbf{x}^{(7)}\right\|_{2}$ & $7.9 \mathrm{e}-147$ & - & - & - & - & - & - & - \\
\hline$p_{c}$ & 2.00 & 8.11 & 2.99 & 4.01 & 4.99 & 6.14 & 7.10 & 8.11 \\
\hline
\end{tabular}

Table 4. Results of TP4 for the $2^{n d} \mathrm{NR}, 8^{\text {th }}$ BQIM with new families of I.F.s $(\mathrm{r}=1,2,3,4,5,6)$

methods (3.1), $r=1,2, \ldots, 6$ to locate the approximate solutions of eq. (4.5). In Table 4, shows that as the convergence order of the algorithm increase the methods converge in less number of iterations with least error. By analysis TP4 problem, our method $8^{\text {th }} \mathrm{KM}$ is converge with least error than $2^{n d} \mathrm{NR}$ and $8^{\text {th }} \mathrm{BQIM}$. Hence, Proposed methods are found to be superior to the methods of $2^{\text {nd }} \mathrm{NR}$ and $8^{\text {th }} \mathrm{BQIM}$.

\section{Application}

\subsection{1-D Bratu Problem}

The one dimensional Bratu problem [6] is given by

$$
\frac{d^{2} U}{d x^{2}}+\lambda \exp U(x)=0, \lambda>0,0<x<1,
$$

with boundary conditions $U(0)=U(1)=0$. The 1-D Bratu problem has two known, bifurcated, actual solutions for values of $\lambda\left\langle\lambda_{c}\right.$, one solution for $\lambda=\lambda_{c}$ and no solution for $\lambda>\lambda_{c}$.

The critical value of $\lambda_{c}$ is simply $8\left(\eta^{2}-1\right)$, where $\eta$ is the fixed point of the hyperbolic cot function $\operatorname{coth}(x)$. The exact solution to equation (5.1) is known and can be presented here as

$$
U(x)=-2 \ln \left[\frac{\cosh \left(x-\frac{1}{2}\right) \frac{\theta}{2}}{\cosh \left(\frac{\theta}{4}\right)}\right],
$$

where $\theta$ may be a constant to be determined, that satisfies the boundary conditions and is fastidiously chosen and assumed to be the answer of the equation (5.1). Employing a similar procedure as in [17], we tend to show the way to acquire the crucial worth of $\lambda$. Substitute equation (5.2) in equation (5.1), simplify and collocate at the point $x=\frac{1}{2}$ because its the centre of the interval. Selected another point, however low order of approximations are probably to being higher suppose the collocation points are distributed somewhat equally throughout the region. Then, we have

$$
\theta^{2}=2 \lambda \cosh ^{2}\left(\frac{\theta}{4}\right)
$$

Differentiating equation (5.3) with respect to $\theta$ and setting $\frac{d \lambda}{d \theta}=0$, the critical value $\lambda_{c}$ satisfies

$$
\theta=\frac{1}{2} \lambda_{c} \cosh \left(\frac{\theta}{4}\right) \sinh \left(\frac{\theta}{4}\right) .
$$

By eliminating $\lambda$ from equations (5.3) and (5.4), we have the value of $\theta_{c}$ for the critical $\lambda_{c}$ satisfying

$$
\frac{\theta_{c}}{4}=\operatorname{coth}\left(\frac{\theta_{c}}{4}\right)
$$

for which $\theta_{c}=4.798714560$ can be obtained using an iterative method. We then get $\lambda_{c}=3.513830720$ from Equation (5.3). Figure 1 illustrates this critical value of $\lambda$. The finite dimensional problem using standard finite difference 


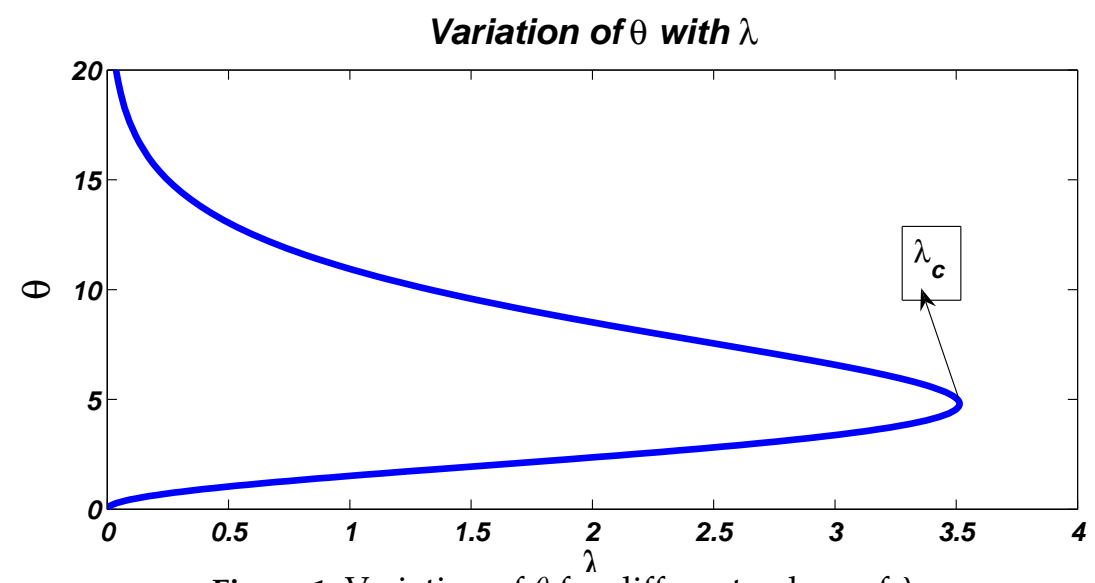

Figure 1. Variation of $\theta$ for different values of $\lambda$.

scheme is given by

$$
F_{j}\left(U_{j}\right)=\frac{U_{j+1}-2 U_{j}+U_{j-1}}{h^{2}}+\lambda \exp U_{j}=0, j=1 . . N-1
$$

with distinct boundary conditions $U_{0}=U_{N}=0$ and also the step size $h=1 / N$. There are square measure $N-1$ unknowns $(n=N-1)$. The Jacobian may be a sparse matrix and its typical range of nonzero per row is 3 . Its noted that the finite difference scheme converges to the lower solution of the 1-D Bratu using the initial vector $U^{(0)}=(0,0, . ., 0)^{T}$.

We use $N=101(n=100)$ and check for $350 \lambda^{\prime}$ s in the interval $(0,3.5]$ (interval breadth $\left.=0.01\right)$. For every $\lambda$, we let $M_{\lambda}$ be the minimum number of iterations for which $\left\|U_{j}^{(k+1)}-U_{j}^{(k)}\right\|_{2}<1 e-13$, where the approximation $U_{j}^{(k)}$ is calculated correct to fourteen decimal places. Let $\overline{M_{\lambda}}$ be the mean of iteration number for the $350 \lambda^{\prime}$ s.

Table 5. Comparison of number of $\lambda^{\prime}$ s (out of $350 \lambda^{\prime}$ s) converging for 1-D Bratu problem

\begin{tabular}{c|ccccc|c}
\hline Method & $M=2$ & $M=3$ & $M=4$ & $M=5$ & $M>5$ & $\overline{M_{\lambda}}$ \\
\hline $2^{\text {nd }} \mathrm{NR}$ & 0 & 12 & 114 & 143 & 81 & 4.92 \\
$8^{\text {th }}$ BQIM & 4 & 257 & 86 & 2 & 1 & 3.26 \\
$3^{\text {rd } K M}$ & 1 & 139 & 170 & 35 & 5 & 3.73 \\
$4^{\text {th }} \mathrm{KM}$ & 4 & 231 & 104 & 10 & 1 & 3.35 \\
$5^{\text {th }} \mathrm{KM}$ & 4 & 250 & 90 & 6 & 0 & 3.28 \\
$6^{\text {th }} \mathrm{KM}$ & 4 & 254 & 88 & 1 & 3 & 3.27 \\
$7^{\text {th }} \mathrm{KM}$ & 4 & 256 & 87 & 1 & 2 & 3.26 \\
$8^{\text {th } K M}$ & 4 & 256 & 87 & 1 & 2 & 3.27 \\
\hline
\end{tabular}

Table 5 give the results for the 1-D Bratu problem, where $M$ denoting number of iterations for convergence. It can be observed from the methods considered in Table 5, proposed methods are most efficient methods than $2^{\text {nd }} \mathrm{NR}$ method, because it has the lowest mean iteration number.

\subsection{2-D Bratu problem}

We consider the solution of the Bratu problem in two-dimensions

$$
\frac{\partial^{2} U}{\partial x^{2}}+\frac{\partial^{2} U}{\partial y^{2}}+\lambda \exp (U)=0, x, y \in D=[0,1] \times[0,1]
$$

subject to the boundary conditions

$$
U(x, y)=0, x, y \in D .
$$

The 2-D Planar Bratu problem has 2 known, bifurcated, actual solutions for values of $\lambda<\lambda_{c}$, one solution for $\lambda=\lambda_{c}$ and no solutions for $\lambda>\lambda_{c}$. The exact solution to eq. (5.7) is known and can be presented here as

$$
U(x, y)=2 \ln \left[\frac{\cosh \left(\frac{\theta}{4}\right) \cosh \left(\left(x-\frac{1}{2}\right)\left(y-\frac{1}{2}\right) \theta\right)}{\cosh \left(\left(x-\frac{1}{2}\right)\left(\frac{\theta}{2}\right)\right) \cosh \left(\left(y-\frac{1}{2}\right)\left(\frac{\theta}{2}\right)\right)}\right],
$$




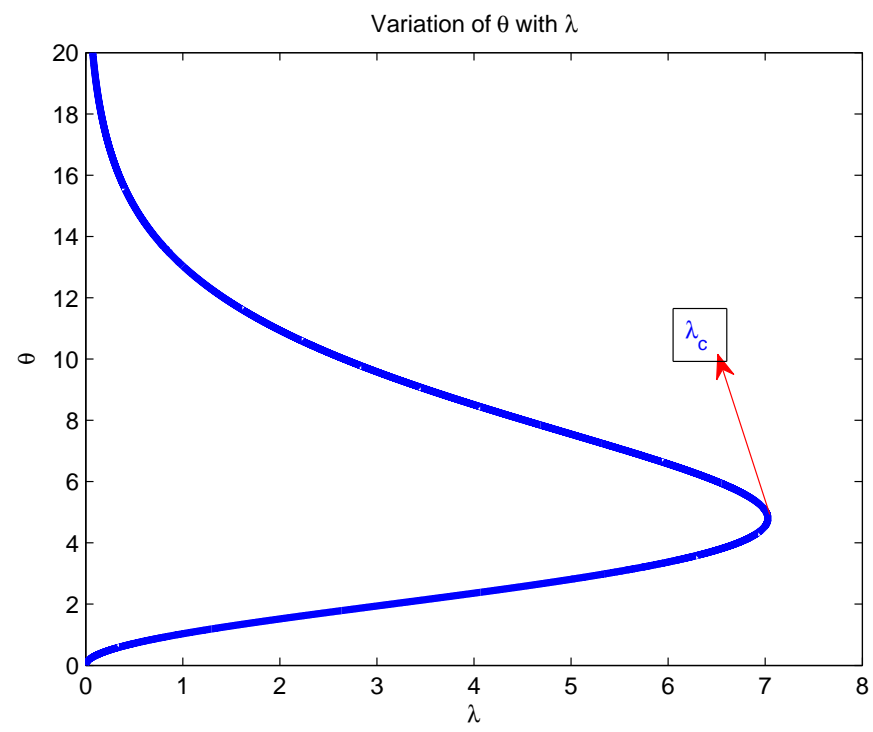

Figure 2. Variation of $\theta$ for different values of $\lambda$.

where $\theta$ may be a constant and to be determined, which satisfies the boundary conditions and is carefully chosen and assumed to be the solution of the equation (5.7). The following procedure found in [17], for how to obtain the critical value of $\lambda$. Substituting eq. (5.9) in (5.7), simplifying and collocating at the point $x=\frac{1}{2}$ and $y=\frac{1}{2}$ because it is the midpoint of the interval. Another point could be chosen, but low-order approximations are likely to be better if the collocation points are distributed somewhat evenly throughout the region. Then, we have

$$
\theta^{2}=\lambda \cosh ^{2}\left(\frac{\theta}{4}\right)
$$

Differentiating eq. (5.10) with respect to $\theta$ and setting $\frac{d \lambda}{d \theta}=0$, the critical value $\lambda_{c}$ satisfies

$$
\theta=\frac{1}{4} \lambda_{c} \cosh \left(\frac{\theta}{4}\right) \sinh \left(\frac{\theta}{4}\right)
$$

By eliminating $\lambda$ from eqs.(5.10) and (5.11), we have the value of $\theta_{c}$ for the critical $\lambda_{c}$ satisfying

$$
\frac{\theta_{c}}{4}=\operatorname{coth}\left(\frac{\theta_{c}}{4}\right)
$$

and $\theta_{c}=4.798714561$. We then get $\lambda_{c}=7.027661438$ from eq. (5.11). Fig. (2) illustrates this critical value of $\lambda_{c}$. The differential equation (5.7) is usually discretized by using the finite-difference five-point formula with the step size $h$, the resulting nonlinear equations are

$$
F\left(U_{i, j}\right)=-\left(4 U_{i, j}-\lambda h^{2} \exp \left(U_{i, j}\right)\right)+U_{i+1, j}+U_{i-1, j}+U_{i, j+1}+U_{i, j-1}
$$

where $U_{i, j}$ is $U$ at $\left(x_{i}, y_{j}\right), x_{i}=i h, y_{j}=j h, i, j=1,2, \ldots N$. Equation (5.13) represents a set of $N \times N$ nonlinear equations in $U_{i, j}$ which are then solved by using iterative methods. We use $N=10$ and $N=20$ for test $700 \lambda^{\prime}$ 's in the interval $(0,7]$ (interval width $=0.01$ ). For each $\lambda$, we let $M_{\lambda}$ be the minimum number of iterations for which $\left\|U_{i, j}^{(k+1)}-U_{i, j}^{(k)}\right\|_{2}<1 e-11$, where the approximation $U_{i, j}^{(k)}$ is calculated correct to 14 decimal places. Let $\overline{M_{\lambda}}$ be the mean of iteration number for the $700 \lambda^{\prime}$ s.

Tables 6, 7 gives the results for 2-D Bratu problem. It can be observed from the proposed methods are considered in Table 6, 7, all the grid points are convergent in two and three iterations only. The proposed methods are most efficient methods than $2^{\text {nd }} N R$, in both the case $N=10$ and $N=20$ because it has the lowest mean iteration number. 
Table 6. Comparison of number of $\lambda$ 's for 2-D Bratu problem for $\mathrm{N}=10$

\begin{tabular}{c|cccc|c}
\hline Method & $M=2$ & $M=3$ & $M=4$ & $M=5$ & $\overline{M_{\lambda}}$ \\
\hline $2^{\text {nd }} N R$ & 0 & 101 & 520 & 79 & 3.96 \\
$8^{\text {th }} B Q I M$ & 43 & 657 & 0 & 0 & 2.93 \\
$3^{r d} K M$ & 18 & 606 & 76 & 0 & 3.08 \\
$4^{\text {th }} K M$ & 43 & 657 & 0 & 0 & 2.93 \\
$5^{\text {th }} K M$ & 43 & 657 & 0 & 0 & 2.93 \\
$6^{\text {th }} K M$ & 43 & 657 & 0 & 0 & 2.93 \\
$7^{\text {th }} K M$ & 43 & 657 & 0 & 0 & 2.93 \\
$8^{\text {th }} K M$ & 43 & 657 & 0 & 0 & 2.93 \\
\hline
\end{tabular}

Table 7. Comparison of number of $\lambda^{\prime}$ s for 2-D Bratu problem for $\mathrm{N}=20$

\begin{tabular}{c|cccc|c}
\hline Method & $M=2$ & $M=3$ & $M=4$ & $M=5$ & $\overline{M_{\lambda}}$ \\
\hline $2^{\text {nd }} N R$ & 1 & 212 & 487 & 0 & 3.69 \\
$8^{\text {th }} B Q I M$ & 89 & 611 & 0 & 0 & 2.87 \\
$3^{\text {rd } K M}$ & 35 & 665 & 0 & 0 & 2.95 \\
$4^{\text {th }} K M$ & 89 & 611 & 0 & 0 & 2.87 \\
$5^{\text {th }} K M$ & 89 & 611 & 0 & 0 & 2.87 \\
$6^{\text {th }} K M$ & 89 & 611 & 0 & 0 & 2.87 \\
$7^{\text {th }} K M$ & 89 & 611 & 0 & 0 & 2.87 \\
$8^{\text {th }} K M$ & 89 & 611 & 0 & 0 & 2.87 \\
\hline
\end{tabular}

\section{Conclusion}

In this work, we have developed a family of iterative methods with three functions evaluations reaching convergence order more than 4 for solving scalar quadratic equations. Furthermore, we have a tendency to show that it is possible to develop methods with same function evaluations of order $r+2$. Also, we have extended these methods to systems for solving quadratic equations. We have showed that Kung-Traub's conjecture fails for quadratic functions. The most advantages of the proposed schemes are the following to solve systems: (i) they are doing not use second order Frechet derivative, (ii) measure only one inverse of first order Frechet derivative and (iii) measure less number of linear systems per iteration. The proposed new methods and their theoretical results are validated through examples whose results are tabulated which gives better results among compared existing methods. For practical applications, the new methods are verified on 1-D, 2-D Bratu problems which gives encouraging results compared to existing methods.

Acknowledgments: I would like to thank the editor and referees for their valuable comments and suggestions. Also, the authors would like to be thankful to the Prof. Dr. R. Ramesh (Principal) and Prof. J. Joy Priscilla (HOD), Saveetha Engineering College, Chennai for their constant encouragement.

\section{References}

[1] Ahmad, F., Comment on: On the kung-traub conjecture for iterative methods for solving quadratic equations. Algorithms, 9:30, 2016.

[2] Babajee, D. K. R. , Madhu, K. and Jayaraman, J., A family of higher order multi-point iterative methods based on power mean for solving nonlinear equations. Afr. Mat., 27(5):865-876, 2016.

[3] Babajee, D.K.R., On the kung-traub conjecture for iterative methods for solving quadratic equations. Algorithms, 9:1, 2016.

[4] Babajee, D.K.R., Madhu, K. and Jayaraman, J., On some improved harmonic mean newton-like methods for solving systems of nonlinear equations. Algorithms, 8:895-909, 2015.

[5] Babajee, D.K.R. and Madhu, K., Comparing two techniques for developing higher order two-point iterative methods for solving quadratic equations. SeMA Journal, Doi: 10.1007/s40324-018-0174-0, 2018. 
[6] Buckmire, R., Investigations of nonstandard Mickens-type finite-difference schemes for singular boundary value problems in cylindrical or spherical coordinates. Num. Meth. P. Diff. Eqns., 19(2):380-398, 2003.

[7] Chun, C. and Kim, Y.I., Several new third-order iterative methods for solving nonlinear equations. Acta Appl. Math., 109:1053-1063, 2010.

[8] Cordero, A., Hueso, J.L., Martinez, E. and Torregrosa, J.R., Accelerated methods of order $2 p$ for systems of nonlinear equations. J. Comp. Appl. Math., 53(4):485-495, 2010.

[9] Cordero, A., Hueso, J.L., Martinez, E. and Torregrosa, J.R., A modified newton-jarratt's composition. Numer. Algor., 55:87-99, 2010.

[10] Cordero, A and Torregrosa, J.R., Variants of Newton's method using fifth-order quadrature formulas. Appl. Math. Comp., 190:686-698, 2007.

[11] Darvishi, M.T. and Barati, A., Super cubic iterative methods to solve systems of nonlinear equations. Appl. Math. Comp., 188:1678-1685, 2007.

[12] Jarratt, P., Some efficient fourth order multipoint methods for solving equations. BIT, 9:119-124, 1969.

[13] Kung, H.T. and Traub, J.F., Optimal order of one-point and multipoint iteration. J. Assoc. Comput. Mach., 21(4):643-651, 1974.

[14] Madhu, K., Note on the kung-traub conjecture for traub-type two-point iterative methods for quadratic equations. International Journal of Advance in Mathematics, Volume 2018(2):112-118, 2018.

[15] Madhu, K., Some new higher order multi-point iterative methods and their applications to differential and integral equation and global positioning system. PhD thesis, Pndicherry University, June 2016.

[16] Madhu, K. and Jayaraman, J., Higher order methods for nonlinear equations and their basins of attraction. Mathematics, 4:22, 2016.

[17] Odejide, S.A. and Aregbesola, Y.A.S., A note on two dimensional Bratu problem. Kragujevac J. Math., 29:49-56, 2006.

[18] Ostrowski, A.M., Solutions of Equations and System of equations. Academic Press, New York, 1960.

[19] Petkovic, M.S., Neta, B., Petkovic, L.D. and Dzunic, J., Multipoint Methods for Solving Nonlinear Equations. Elsevier, Amsterdam, 2013.

[20] Sharma, J.R. and Arora, H., An efficient family of weighted-newton methods with optimal eighth order convergence. Appl. Math. Lett., 29:1-6, 2014.

[21] Thukral, R., New modification of newton method with third-order convergence for solving nonlinear equations of type $f(0)=0$. American Journal of Computational and Applied Mathematics, 6(1):14-18, 2016.

[22] Wait, R., The Numerical Solution of Algebraic Equations. John Wiley and Sons, 1979.

\section{Affiliations}

\section{KALYANASUNDARAM MADHU}

AdDress: Department of Mathematics, Saveetha Engineering College, Chennai - 602105, India.

E-MAIL: kalyan742@pec.edu

ORCID ID: 0000-0002-9328-2996 